\title{
The strings connection: MSSM-like models from strings
}

\author{
Hans Peter Nilles ${ }^{\mathrm{a}}$ \\ Bethe Center for Theoretical Physics (BCTP) and Physikalisches Institut der Universität Bonn, Nussallee 12, 53115 Bonn, Germany
}

Received: 20 November 2013 / Accepted: 27 November 2013 / Published online: 27 May 2014

(C) The Author(s) 2014. This article is published with open access at Springerlink.com

\begin{abstract}
String theory constructions towards the MSSM allow us to identify some general properties that could be relevant for tests at the LHC. They originate from the geometric structure of compactification and the location of fields in extra-dimensional space. Within the framework of the heterotic MiniLandscape we extract some generic lessons for supersymmetric model building. Among them is a specific pattern of SUSY breakdown based on mirage mediation and remnants of extended supersymmetry. This leads to a split spectrum with heavy scalars of the first two families of quarks and leptons and suppressed masses for gauginos, top partners and Higgs bosons. The models exhibit some specific form of hidden supersymmetry consistent with the high mass of the Higgs boson and all presently available experimental constraints. The most compelling picture is based on precision gauge coupling unification that might be in the kinematic reach of the LHC.
\end{abstract}

\section{Introduction}

The standard model (SM) of particle physics and its minimal supersymmetric extension (MSSM) are under experimental investigation at the Large Hadron Collider (LHC) and other high energy physics experiments. The recent discovery of the Higgs boson $[1,2]$ has strong impact on the SM and the MSSM. While these models were constructed to understand particle physics at the TeV scale from a bottomup perspective, it would be of interest to extend them to higher energies and find a consistent ultraviolet (UV) completion. The UV extrapolation of the MSSM seems to be consistent with a grand unified (GUT) picture where all the gauge interactions are derived from a unified group structure. The inclusion of gravitational interactions would point to a UV-completion within superstring theory. In such a unified scheme we would then hope for a better under-

\footnotetext{
a e-mail: nilles@th.physik.uni-bonn.de
}

standing of the many free parameters of the SM and the MSSM.

The construction of particle physics models from string theory started with great enthusiasm in the mid-1980s triggered by the seminal paper of Green and Schwarz [3], suggesting unified gauge groups like $\mathrm{SO}(32)$ and $E_{8} \times E_{8}$. Meanwhile we can look back at model constructions within the framework of heterotic theories, type I, type IIA and B as well as M- and F-theory. ${ }^{1}$

Unfortunately string theories do not lead exclusively to the SM or MSSM at low energies. These models are not a generic part of the so-called "Landscape" of string theories. To find them (if at all) we have to look at specific spots and corners of this landscape. The task of string phenomenology is thus an effort to see whether the MSSM can be embedded in string theory (rather than derive it directly from string theory). Once such embeddings are found we can then try to extract common properties shared by the successful models as possible "predictions" of string theory. Relevant issues concern gauge-Yukawa unification, gauge-Higgs unification, a solution to the $\mu$-problem, the flavor structure and the absence of exotics.

String theory is defined in $d=10$-dimensional space-time while SM and MSSM reside in $d=4$. Properties of the $d=4$ models depend crucially on the compactification of the extra spatial dimensions. Here it is not only the geometry of the compact manifold, but also the geographic localization of the fields on that manifold. Essentially all low-energy physics is given by these geometrical and geographical properties. This includes the possible appearance of scale hierarchies within the framework of string theories. They could come from locations of enhanced symmetry at corners of the moduli space of the extra-dimensional manifold. String model building is thus a map of the "Landscape" of extra dimensions to the "Landscape" of string vacua with SM or MSSM structure. Such an analysis requires the construction of a vast amount

\footnotetext{
${ }^{1}$ For recent reviews and a comprehensive list of references see [4-8].
} 
of possible string vacua followed by a selection of acceptable MSSM candidates.

Given the apparently enormous amount of string vacua we have to establish some useful rules where to look first. In Sect. 2 we shall discuss these rules followed by a discussion of the compactification in Sect. 3. This will lead to the construction of the so-called Minilandscape [9-11] in Sect. 4. In Sect. 5 we shall extract lessons from the Minilandscape, most notably for the Higgs system, the top-quark Yukawa coupling, the flavor symmetries and a specific pattern of supersymmetric breakdown. Explicit model building towards tests at the LHC will be treated in Sect. 6. In Sect. 7 we shall give a conclusion and outlook.

\section{Some useful rules}

The MSSM has special properties that point towards a specific UV-completion. We have the gauge group $\mathrm{SU}(3) \times$ $\mathrm{SU}(2) \times U(1)$, three families of quarks and leptons and one pair of Higgs doublets. Evolution of gauge coupling constants shows unification at a scale of few times $10^{16} \mathrm{GeV}$. Neutrino mass spectra are consistent with a see-saw mechanism that requires a right handed neutrino with Majorana mass at a similar scale. One family of quarks and leptons fits exactly into one 16-dimensional spinor representation of $\mathrm{SO}(10)$. The families come in three identical repetitions. $\mathrm{SO}(10)$ (and its subgroups $\mathrm{SU}(5)$ or $\mathrm{SU}(4) \times \mathrm{SU}(2) \times \mathrm{SU}(2))$ represents the most economical GUT-extension of $\mathrm{SU}(3) \times \mathrm{SU}(2) \times U(1)$. While quarks and leptons come in complete representations of the GUT group, the Higss multiplet is incomplete (known as the doublet-triplet splitting problem in the SU(5) framework). Apart from the gauge symmetries the MSSM contains many more (approximate) discrete symmetries to explain the flavor structure and the stability of the proton.

This leads us to postulate the following rules for the embedding of the SM in string theory $[12,13]$ :

- include spinor representations of $\mathrm{SO}(10)$ for chiral matter multiplets and the description of quarks and leptons,

- allow simultaneously for split GUT-multiplets in case of Higgs fields (to solve the "doublet-triplet splitting" problem),

- repetition of families does not come from an enlarged gauge group but is understood as a result of the topological properties of compactified space,

- consider $N=1$ supersymmetry in $d=4$ to allow for gauge coupling unification

- select specific corners of moduli space (of the compact manifold) to allow for enhanced discrete symmetries.
From the string theory point of view we can also deduce some tendency towards a grand unified picture, where in particular $E_{8}$ plays a crucial role. $E_{8}$ is the largest exceptional group.

In $d=4$ it does not allow for chiral fermion representations. This is different in $d=10$ and $E_{8}$ is a valid GUT group provided the symmetry is broken during the process of compactification. But how does this connect to the successful grand unified gauge groups $\mathrm{SU}(5)$ and/or $\mathrm{SO}(10)$. There is a well-defined chain to descent from $E_{8}$ to smaller groups by chopping off a node of the Dynkin diagram. This leads to $E_{7}$, then to $E_{6}$ and $E_{5} . E_{5}$ is not an exceptionalgroup: it

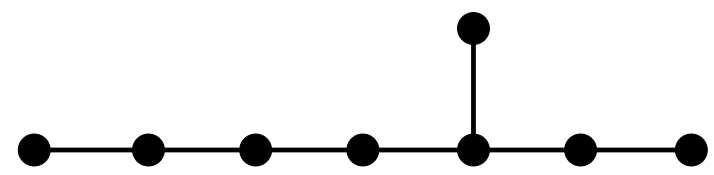

Fig. $1 E_{8}$ is the maximal exceptional group

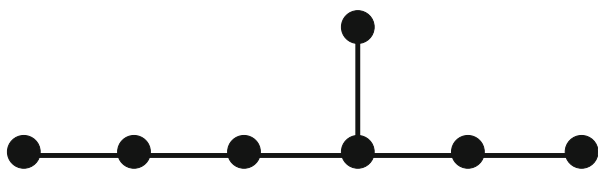

Fig. 2 The next smaller is $E_{7}$

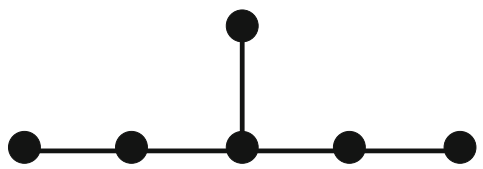

Fig. $3 E_{6}$ allows for chiral representation in $d=4$

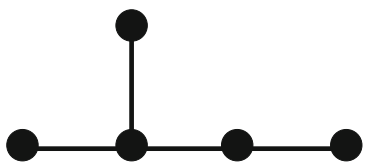

Fig. $4 E_{5}$ coincides with $D_{5}=\mathrm{SO}(10)$

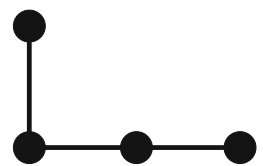

Fig. $5 E_{4}$ is equivalent to $A_{4}=\mathrm{SU}(5)$

Fig. $6 E_{3}$ connects to the standard model gauge group 
coincides with $D_{5}=\mathrm{SO}(10)$. Further steps in the chain are $E_{4}=A_{4}=\mathrm{SU}(5)$ and $E_{3}=\mathrm{SU}(3) \times \mathrm{SU}(2)$ makes contact to the real world (the breakdown of the symmetries in terms of Dynkin diagrams is illustrated in Figs. 1, 2, 3, 4, 5, 6).

While $E_{8}$ looks esoteric from the point of view of the lowenergy effective field theory it appears naturally in the framework of string theory. This is obvious for the $E_{8} \times E_{8}$ heterotic string, but we can also find $E_{8}$ as a nonperturbative enhancement in M- and F-theory. Since the latter two only have a qualitative description at strong coupling, we shall concentrate here on a discussion in the framework of heterotic $E_{8} \times E_{8}$ string $[14,15]$, where a fully consistent global string construction is possible. Even here the MSSM is not generic but will require the selection of special corners in moduli space with enhanced discrete symmetries. Some remnants of grand unification and spinors of $\mathrm{SO}(10)$ will be present as well.

\section{The process of compactification}

Celebrated examples for compactification to $d=4$ with $N=1$ supersymmetry [16] are Calabi-Yau manifolds. They are beautiful objects (see Fig. 7) but quite difficult to construct. While we can make some reliable statements on topological properties, a more detailed description of the metric is barely possible. In addition Calabi-Yau compactification gives a "generic" description of moduli space and we might miss some of the "corners" of moduli space that are relevant for the appearance of small parameters in the low-energy effective action.

We thus need an approximation that is simple enough to allow for specific calculations in the framework of string theory. In addition it should encode all the topological properties

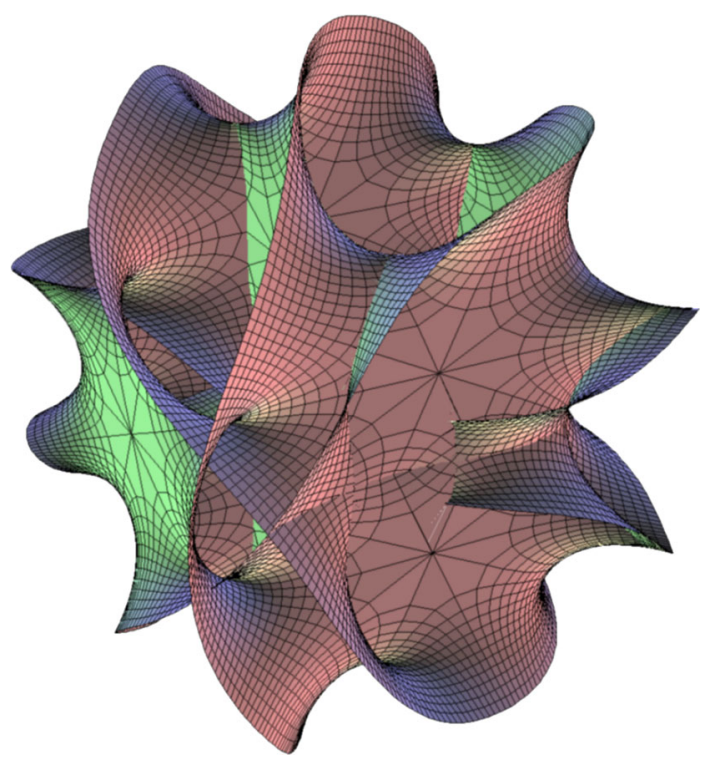

Fig. 7 An impression of a Calabi-Yau manifold

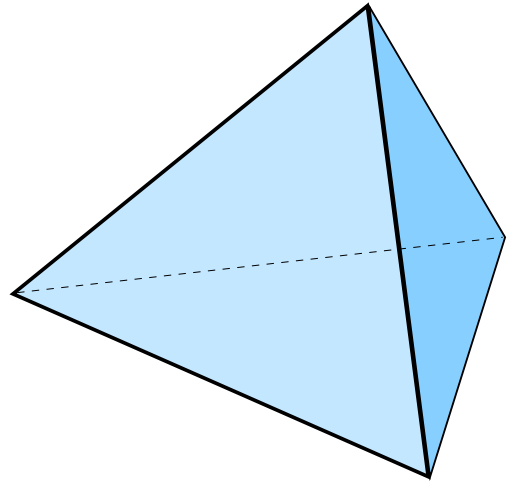

Fig. 8 A two-dimensional $Z_{2}$ orbifold

of the Calabi-Yau manifold and provide an intuitive geometrical picture of the location of fields in extra dimensions. In our approach we shall use the notion of (flat) orbifold compactifications [17-20] of the $E_{8} \times E_{8}$ heterotic string. ${ }^{2}$

Orbifolds are spatially flat objects with the exception of fixed points (fixed tori) where curvature contributions are localized (see Fig. 8 for an illustration). The flatness allows an exact treatment in the framework of conformal field theory and the geometric picture is simple and intuitive. We encounter several sectors that characterize the location of fields in extra dimensions:

$-d=10$ untwisted sector with fields traveling throughout the six compactified dimensions (bulk)

$-d=6$ twisted sector (fixed tori in compactified extra dimensions)

$-d=4$ twisted sector (fixed points in extra dimensions)

In addition there is also a "localization" of gauge fields in extra dimensions in the sense that we find different effective gauge groups at different loci of fields. To illustrate this we shall consider an explicit example discussed in [13] (see Fig. 9). We find different manifestations of gauge symmetry at different fixed points. The fields located at the fixed points will come with the representations of that group. So if the electron lives at the $\mathrm{SO}(10)$ fixed point it will come in a full spinor representation of $\mathrm{SO}(10)$. At other points we might have split representations with respect to $\mathrm{SO}(10)$. So the Higgs fields should not be localized at an $\mathrm{SO}(10)$ fixed point. Otherwise they would come in a full 10-dimensional representation of $\mathrm{SO}(10)$ and there would be $\mathrm{SU}(3)$ triplets in addition to the desired SU(2) doublets of the MSSM. The $d=4$ gauge group is the common subgroup of the gauge groups at the various fixed points, here the SM gauge group $\mathrm{SU}(3) \times \mathrm{SU}(2) \times U(1)$ (see Fig. 10).

This leads to a picture called "Local Grand Unification". In $d=4$ the gauge group is $\mathrm{SU}(3) \times \mathrm{SU}(2) \times U(1)$ while

\footnotetext{
${ }^{2}$ For other approaches see [21-31].
} 


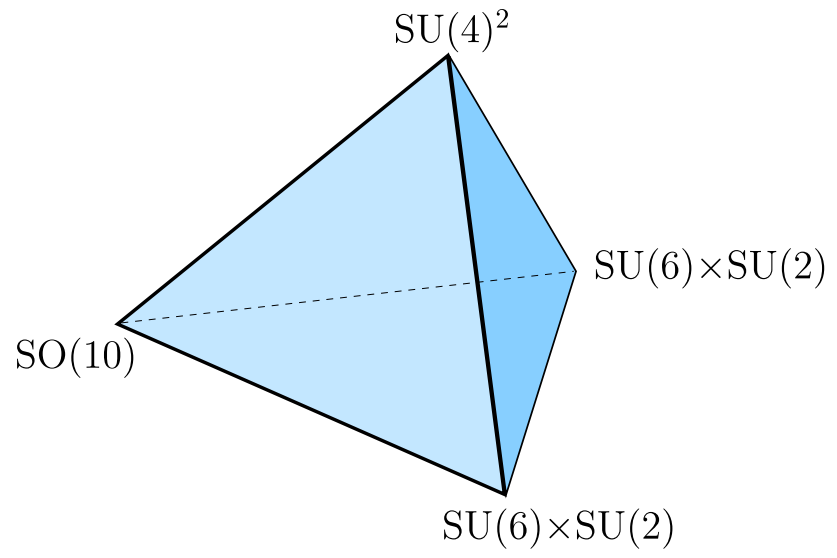

Fig. 9 The gauge symmetries at the various fixed points for a specific model constructed in Ref. [13]

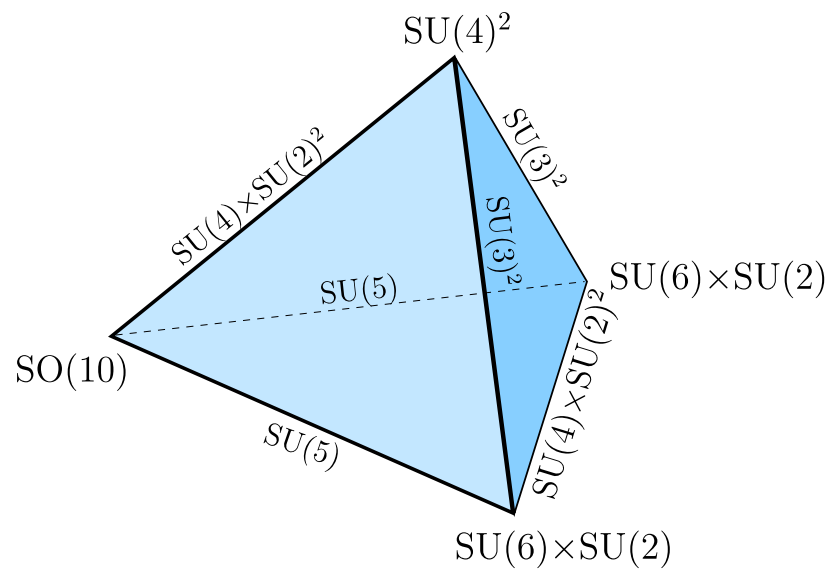

Fig. 10 The gauge symmetries at the various fixed points and their common subgroups. In the present model this leads to the standard model gauge group in the four-dimensional effective theory [13]

the gauge symmetry is enhanced at certain fixed points (fixed tori) in extra dimensions. The scheme keeps the good properties of grand unification like the complete GUT family structure for quarks and leptons. It avoids the problems like complicated $d=4$ GUT breaking and the doublet-triplet splitting problem via the appearance of split multiplets. It is obvious that in such a picture the key properties of the $d=4$ effective theory will depend crucially on the geography of fields in extra dimensions.

\section{A MiniLandscape}

To complete this program we have to construct explicit models of MSSM structure. With a sufficiently large sample of such models we should then be able to identify "fertile" patches of the string landscape and analyse the successful models to check for regularities. A first attempt to do so was an analysis of heterotic $E_{8} \times E_{8}$ models within the orbifold compactification $Z_{6} I I$. We will not repeat the details here, as they are explained in [9-11,32-35]. Meanwhile these investigations have been extended to the $Z_{2} \times Z_{2}$ case $[13,36], Z_{12}$ [37,38], $Z_{2} \times Z_{4}$ [39] and $Z_{8}$ [40]. All these constructions are based on a grand unified picture at some intermediate stage. This is one of the reasons for their success. If you start with a grand unified picture it is much more likely to find successful models. Partially this comes from the fact that one family of quarks and lepton fits into the 16-dimensional spinor representation of $\mathrm{SO}(10)$ as we have discussed in Sect. 2.

As we have stressed before, we expect the geometry and the localization of fields in compactified dimensions to be important for properties of the low-energy MSSM effective action. Therefore we need models that are not only consistent vacua of string theory, but we also need explicit information as regards the "geography" of fields in extra dimensions. By now these two properties are only available in the orbifold constructions mentioned above. Let us illustrate this in the case of the $Z_{6} I I$ orbifold. These consist of a $Z_{2}$ twist $\theta$ and a $Z_{3}$ twist $\omega$. We consider the six-dimensional compactified space as a product of three tori as shown in Fig. 11.

The fields on the orbifold can now be associated to specific sectors. First there are the fields in the untwisted sector. These are the fields that exist already on the torus and can therefore freely move through six-dimensional compactified space (so they are bulk fields that actually live in full ten-dimensional space-time). In addition we have twisted fields attached to various sectors. They do not live in the full bulk, but they are confined to fixed points and fixed tori in compactified space. For $Z_{6} I I$ we have three twisted sectors as shown in Figs. 12, 13 and 14, one of them with fixed points and two of them with fixed tori. For a given MSSM-candidate model we shall then be able to identify exactly the location of all the fields. These properties will be important for the interactions between fields in the given model. Fields that live close to each other (or have sufficient overlap) will couple more strongly than those at remote corners of extra-dimensional space.

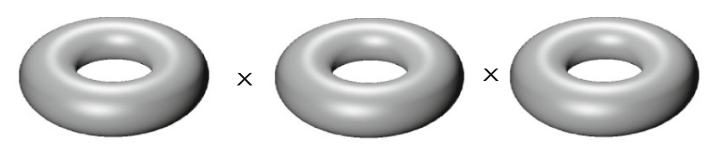

Fig. 11 The bulk of six-dimensional compactified space. Fields in the untwisted sector can freely move in the bulk

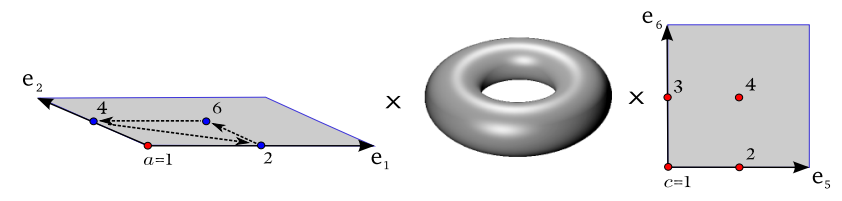

Fig. 12 The sector of $Z_{2}$ twist ( $\theta$ sector) with fixed torus 


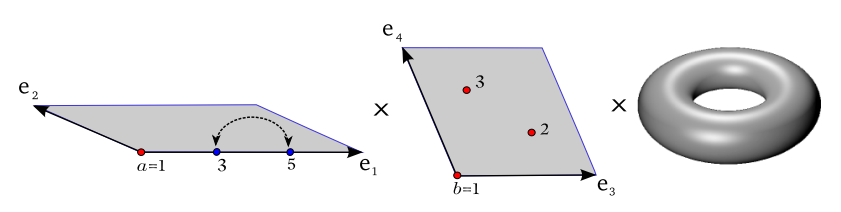

Fig. 13 The sector of $Z_{3}$ twist ( $\omega$ sector) with fixed torus

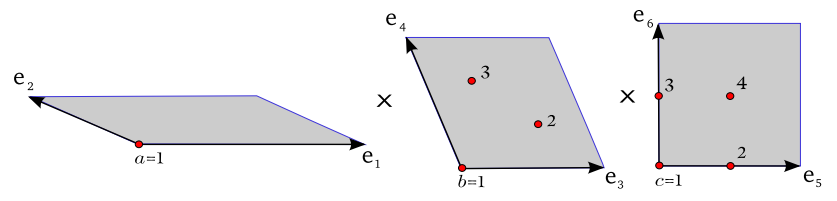

Fig. 14 The sector of $Z_{6}$ twist $(\theta \omega$ sector $)$ with fixed points

The construction principle for the models has been given in refs. [9-11]. One identifies models at the orbifold fixed points with enhanced gauge and discrete symmetries (in this construction we typically find several additional $U(1)$ - symmetries). One of the $U(1)$-symmetries is anomalous and thus induces a nontrivial Fayet-Iliopoulos (FI) term. As a result some singlet fields will develop nontrivial vacuum expectation values that break the additional $U(1)$-symmetries and allow a decoupling of exotics. As there is a small parameter $\epsilon$ involved, ${ }^{3}$ this analysis can be done using effective field theory methods. It requires a scan of F- and D-flat directions in the effective potential. The resulting models will then inherit the symmetries at the orbifold point in a slightly broken form and various hierarchies in the model can be obtained in the spirit of the Frogatt-Nielsen mechanism [41]. This will be instrumental for the discussion of Yukawa couplings of the MSSM. Small parameters appear as powers of $\epsilon$ and are the source for the appearance of mass hierarchies (e.g. the $\mu$-term) in the low-energy effective theory. With a sufficiently large sample of candidate models we can now "experimentally" study the properties of these models and try to extract some lessons for further model building.

\section{Lessons from the MiniLandscape}

All the models have gauge group $\mathrm{SU}(3) \times \mathrm{SU}(2) \times U(1)$ in the observable sector and possibly some hidden sector gauge group which might be relevant for supersymmetry breakdown (which we will not discuss here in detail). They have three families of quarks and leptons and exactly one pair of Higgs doublets $H_{u}$ and $H_{d}$. All potential Higgs triplet pairs are removed and the doublet-triplet splitting problem is solved. Still there remains the question of a possible $\mu H_{u} H_{d}$

\footnotetext{
${ }^{3}$ In the models of the MiniLandscape the parameter $\epsilon$ is typically of the order $1 / 10$ to $1 / 100$.
}

term and we shall therefore start our discussion with the Higgs system.

\subsection{Lesson 1: the Higgs doublets}

The Higgs system is vector-like and a $\mu$-term $\mu H_{u} H_{d}$ is potentially allowed. As this is a supersymmetric term we would like to understand why it is small compared to the GUT scale: This is the so-called $\mu$-problem. To avoid the problem one could invoke a symmetry that forbids the term. However, we know that $\mu$ has to be nonzero and the symmetry has to be broken and this might reintroduce the $\mu$-term again. In string theory the problem is even amplified since typically we find several (say N) Higgs doublet pairs at the orbifold point. In the procedure to remove exotics (as described above) we have to make $N-1$ pairs heavy while keeping one light. So this last doublet has to resist the mechanism that allows to remove the others. In fact in many constructions to "solve" the $\mu$-problem in this way the small $\mu$-parameter is the result of a specific fine tuning of parameters arranged in such a way to remove all doublet pairs except for one, and we do not consider this as a satisfactory solution. Part of the models of the MiniLandscape are in this class, but fortunately only very few.

Most of the models provide one Higgs-doublet pair that resisted all attempts to remove them. At first we were surprised by this result and expected a "hidden" symmetry to be at work. In fact we could identify an underlying discrete R-symmetry [10] that protected the $\mu$-parameter. In some cases this R-symmetry [42-44] was only approximate and could therefore allow a $\mu$-parameter at a higher order in the superpotential sufficiently suppressed by a high power of the Fayet-Iliopoulos parameter $(\epsilon)$.

Where does this come from? Is there a common property in the models that is the source of this astonishing result? Indeed there is! In all of these models the Higgs fields $H_{u}$ and $H_{d}$ live in the untwisted sector! This is quite a special situation: Higgs pairs in $d=4$ come from gauge fields in extra dimensions and this is called "gauge-Higgs unification". Technically the Higgs fields correspond to continuous Wilson lines in the sense described in $[45,46]$. But why does it lead to a solution of the $\mu$-problem? The Higgs pair is nontrivially extended in the bulk. Therefore it might be in a nontrivial representation of the extra-dimensional Lorentz group $\mathrm{SO}(6)$ of $\mathrm{SO}(9,1)$. The group $\mathrm{SO}(6)$ treats bosons and fermions differently and can thus provide the required (discrete) R-symmetry to control the $\mu$-term. This is the first lesson from the MiniLandscape: The Higgs doublets live in the untwisted sector and are thus bulk fields (by now this has been confirmed in other constructions, like $Z_{2} \times Z_{2}[13,36]$ and $Z_{2} \times Z_{4}$ [39]). We believe that this result derived from the MiniLandscape might be of much more general validity. 
The R-symmetry forbids a constant term in the superpotential as well. This has two important consequences

- SUSY vacua are Minkowski vacua with vanishing vacuum energy

- SUSY breakdown requires a nontrivial constant in the superpotential, thus breaks the R-symmetry and relates $\mu$ to the value of the gravitino mass.

The MiniLandscape gives a solution to the $\mu$-problem via an approximate (discrete) R-symmetry. Looking in detail at the models we find that the term $H_{u} H_{d}$ in the superpotential is neutral under all selection rules. Thus if a term $f\left(\Phi_{i}\right) H_{u} H_{d}$ (where $f\left(\Phi_{i}\right)$ is a polynomical of singlet fields) is allowed in the superpotential, a term $f\left(\Phi_{i}\right)$ is allowed as well. This is reminiscent of an earlier discussion of Casas and Munoz [47] in field theoretical models. To repeat the first lesson of the MiniLandscape: Higgs fields live in the untwisted sector, they are bulk fields.

\subsection{Lesson 2: the top quark}

The large mass of the top quark requires a large top-quark Yukawa coupling (in contrast to the Yukawa couplings for the first two families which should be small compared to the gauge couplings). String theory has only one coupling for gauge and Yukawa couplings. A trilinear top-quark Yukawa coupling $H_{u} t \bar{t}$ would be of order of the gauge couplings and thus lead to gauge-Yukawa unification.

In the construction of models of the MiniLandscape we required a Yukawa coupling at the trilinear level to accommodate a heavy top quark. Other Yukawa couplings could be suppressed within the framework of a Frogatt-Nielsen mechanism.

Inspection of the location of the top quark yields a second lesson of the MiniLandscape: both $(\mathrm{t}, \mathrm{b})$ and $\bar{t}$ reside in the untwisted sector as well. This guarantees maximal overlap with the bulk field $H_{u}$ and leads to gauge-Yukawa unification in a natural way. ${ }^{4}$ Typically the top quark is the only matter field with trilinear Yukawa coupling. The location of the other fields of the third family is strongly model-dependent, but in general they are distributed over various sectors: the third family could be called a "patchwork family".

\subsection{Lesson 3: first two families of quarks and leptons}

They are found to be located at fixed points in extra dimensions (Fig. 14). As such they live at points of enhanced symmetries (both gauge and discrete). The presence of these dis-

\footnotetext{
${ }^{4}$ An exception is the $Z_{2} \times Z_{2}$-orbifold, which does not allow for quarks and leptons in the untwisted sector. For a detailed discussion see refs. $[13,36]$.
}

crete symmetries is the reason for the suppressed Yukawa couplings. In the $Z_{6} I I$ example shown in the figure the two families live at adjacent fixed points in the third extradimensional torus. In fact one family is located at $a=$ $b=c=1$ the other at $a=b=1$ and $c=3$ (see Fig. 14). This leads to a $D_{4}$ family symmetry $[48,49]$ that forbids sizeable flavor changing neutral currents and thus relieves the so-called "flavor problem". The geometric reason for small Yukawa couplings is their minimal overlap with the bulk Higgs fields. This leads to Yukawa couplings of higher order and a hierarchical generation of masses within the Frogatt-Nielsen mechanism. The FI-term provides the small parameter $\epsilon$ that controls the pattern of the masses. The first two families also live at points of enhanced gauge symmetries and enjoy the successful properties of "local grand unification".

\subsection{Lesson 4: the pattern of SUSY breakdown}

The question of supersymmetry breakdown is a complicated process and we shall try to extract some general lessons that are rather model-independent. Specifically we would consider gaugino condensation in the hidden sector [50-53] (realized explicitly in the MiniLandscape [54]).

A reasonable value for the gravitino mass can be obtained if the dilaton is fixed at a realistic value for the grand unified gauge coupling $\alpha_{\text {GUT }} \sim 1 / 24$. The discussion needs the study of moduli stabilization, which, fortunately, we do not have to analyse here in detail. In fact we can rely on some specific pattern of supersymmetry breaking which seems to be common in various string theories, first observed in the framework of Type IIB theory [55-61] and later confirmed in the heterotic case [62,63]: so-called "mirage mediation". Its source is a suppression of the tree-level contribution in modulus mediation (in particular for gaugino masses and Aparameters). The suppression factor is given by the logarithm of the "hierarchy"

$\log \left(M_{\text {Planck }} / m_{3 / 2}\right)$

which numerically is of the order $4 \pi^{2}$. Non-leading terms suppressed by loop factors can now compete with the treelevel contribution. In its simplest form the loop corrections are given by the corresponding $\beta$-functions, leading to "anomaly mediation" if the tree-level contribution is absent. The mirage scheme is therefore a combination of modulus and mirage mediation. At the GUT scale soft terms (say gaugino masses) receive a universal contribution from modulus mediation while the contribution of loops splits the spectrum proportional to the $\beta$-function. As the $\beta$-function for SU(3) is negative this leads to a suppressed value of the gluino mass at the GUT scale while the contributions to the wino and bino are increased. As the evolution of couplings to lower ener- 


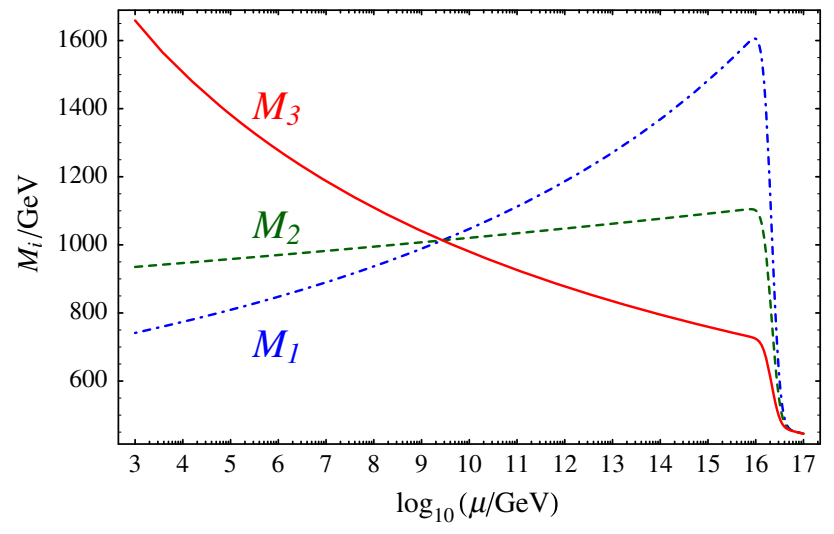

Fig. 15 The evolution of gaugino masses in mirage mediation. The scale of equality of the gaugino masses depends on the ratio of modulus versus anomaly mediation. For details see Ref. [59]

gies is determined by the same $\beta$-functions one is lead to a situation that gaugino masses coincide at some intermediate scale: the mirage point (see Fig.15).

The location of this point depends on the relative strength of modulus and anomaly mediation. It could be as low as the $\mathrm{TeV}$ scale. We can thus summarize the main properties of mirage mediation:

- gaugino masses and A-parameters are suppressed compared to the gravitino mass by the factor $\log \left(M_{\text {Planck }} / m_{3 / 2}\right)$

- we obtain a compressed pattern of gaugino masses (as the $\mathrm{SU}(3) \beta$-function is negative while those of $\mathrm{SU}(2)$ and $U(1)$ are positive)

- soft scalar masses $m_{0}$ are more model-dependent. In general we would expect them to be as large as $m_{3 / 2}$ [57].

This picture of mirage mediation is a quite generic property of string theory and has been explicitly discussed within type IIB and the heterotic string theory. It is a consequence of the mechanism to fine tune the vacuum energy to the observed value.

The models of the MiniLandscape inherit this generic picture of suppressed and compressed gaugino masses and suppressed A-parameters. But they also teach us something new on the soft scalar masses: and this leads to lesson 4 of the MiniLandscape. The scalars (Higgses as well as squarks and sleptons) reside in various sectors that feel SUSY in different ways: some of them enjoy extended SUSY (at the tree level). If we consider the untwisted sector we know that it is obtained from simple torus compactification of the $D=10$ theory and this leads to extended $N=4$ supersymmetry in $D=4$. Soft terms are protected (at least at tree level) by this symmetry (and broken by loop corrections when they communicate to sectors with a smaller amount of supersymmetry). Sectors with fixed tori feel a remnant $N=2$ supersymmetry and might be protected as well. The fields in sectors with fixed points feel only $N=1$ SUSY and are not further protected $[64,65]$. Within the framework described here we would then expect soft terms $m_{0} \sim m_{3 / 2}$ for the first two families. Other scalar fields, in particular the Higgs bosons and the scalar partners of the top quark, feel a protection from extended SUSY and are therefore suppressed compared to $m_{3 / 2}$ (by a loop factor of order $1 / 4 \pi^{2}$ ).

The pattern of soft terms in the models of the MiniLandscape can thus be characterized by two scales: the gravitino mass $m_{3 / 2}$ and a second scale suppressed by a factor of order of $1 / 4 \pi^{2}$. As a result of mirage mediation and the consequences of extended SUSY we see a characteristic hierarchical pattern. Higgs bosons, stops, gaugino masses and Aparameters are suppressed compared to the gravitino mass while squark and sleptons of the first two families are heavy. In addition we expect a compressed spectrum of gaugino masses at the $\mathrm{TeV}$ scale. This constitutes lesson 4 of the MiniLandscape.

\section{Connections to LHC results}

At the moment this is written (summer 2013) we have two important results from the first run of the LHC at center of mass energy $7-8 \mathrm{TeV}$. These are

- the discovery of the Higgs boson at a mass of 125-126 $\mathrm{GeV}$ providing the last missing piece of the standard model

- apparent absence of any sign of physics beyond the standard model

Given the high expectations for new physics signals at LHC both results put severe constraints on the parameter space of the MSSM. One might even ask the question whether this is still compatible with the MSSM. Does the stringinspired scheme we are discussing here survive the experimental results of the LHC?

\subsection{Qualitative string "predictions"}

There is no convincing way known to predict the Higgs mass directly within the models of the MiniLandscape. We have to accommodate it as an experimental fact. Pre-LHC we had the lower limit of $114 \mathrm{GeV}$ for the Higgs mass from the LEP experiments. Within the MSSM we had thus a possible range of $114-130 \mathrm{GeV}$. The string-inspired scheme discussed here can, however, say something about the SUSY spectrum. The two most important results are:

- the spectrum of SUSY particles exhibits a hierarchy of scales separated by the value of $\log \left(M_{\text {Planck }} / m_{3 / 2}\right)$. This implies that the gravitino mass $m_{3 / 2}$ has to be very large, presumably even bigger than $10 \mathrm{TeV}$ or more. Otherwise 
gaugino masses would be too small and already ruled out experimentally. This leads to heavy masses $m_{0} \sim m_{3 / 2}$ for the scalars of the first two generations. This, in addition to the presence of the $D_{4}$ family symmetry, relieves potential tension in the absence of flavor changing neutral currents. Unfortunately we do not have a theoretical upper limit of $m_{0} \sim m_{3 / 2}$ from the MSSM and the stringinspired system. A limitation on $m_{0} \sim m_{3 / 2}$ would need some prejudice concerning the fine tuning one is willing to tolerate.

- a compressed spectrum of gaugino mass as given by the mirage mediation scheme. Recall that in the case of universal gaugino masses at the GUT scale we would have the ration $M_{1}: M_{2}: M_{3} \approx 1: 2: 6$ at the electroweak scale. In the mirage scheme this ratio depends on the relative size of the contributions of modulus mediation and anomaly mediation. The spectrum is typically more compressed even to a point where $M_{1} \approx M_{2} \approx M_{3}$ at the $\mathrm{TeV}$ scale [67].

The compression of the gaugino mass scale has several characteristic consequences:

- missing energy signals at LHC will be less efficient to detect SUSY particles.

- there will be a reduced fine-tuning problem because the gluino mass is suppressed.

- we could achieve precision gauge unification.

- in the presence of an ultra-compressed spectrum we might solve potential problems of the thermal relic abundance of dark matter candidates.

We shall discuss these issues in detail later when we consider explicit models.

\subsection{Lessons from LHC}

The value of the Higgs mass is compatible with the MSSM but hints to a rather high value of masses of SUSY particles (see Fig.16).

We are thus driven to a corner of parameter space with large SUSY scale $M_{\text {SUSY }}$. For this reason there are already many attempts in the literature to build models beyond the MSSM. Here we shall stick to the discussion of the MSSM since we do not think that the absence of experimental signals for physics beyond the SM, at this moment, is a sufficient motivation to go beyond the MSSM.

In fact we note that the high value of the Higgs mass leads to high $M_{\text {SUSY }}$ in the MSSM. It should therefore be not too surprising that LHC has not found signs of SUSY in the first run. $M_{\text {SUSY }}$ might be too large for supersymmetry to be just

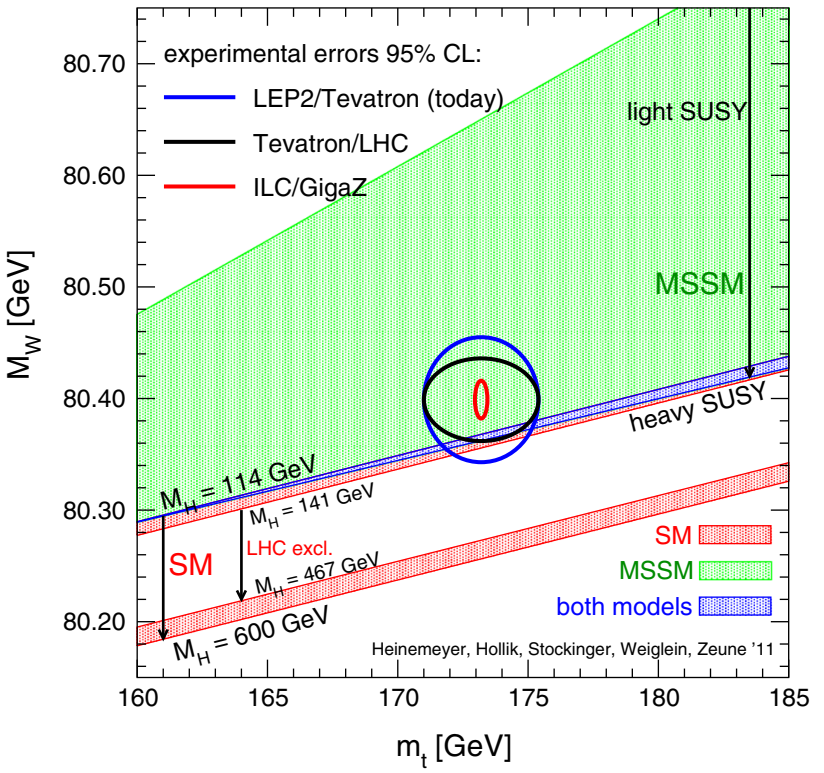

Fig. 16 Constraints on the SUSY spectrum [66] from LEP. In the MSSM a Higgs mass of $126 \mathrm{GeV}$ leads to the region of "heavy SUSY"

around the corner and one might even fear that the energy reach of LHC14 might not be sufficient.

\subsection{Towards explicit models}

We have the parameters $m_{3 / 2}$ and $m_{0}$ in the multi-TeV range, while other parameters like $m_{3}$ (stops), A and gaugino masses $\left(m_{1 / 2}\right)$ are suppressed by a factor of $O\left(1 / 4 \pi^{2}\right)$ and could be accessible at the LHC. An important role is played by the mirage parameter $\varrho$, which gives the ratio of modulus to anomaly mediation for gaugino masses. It defines the compression of the spectrum of gaugino masses. Model building along these lines has been discussed explicitly in refs. $[64,65]$. We shall here only explain the outcome and skip the details. Not surprisingly, the high value of the Higgs mass limits the parameter space drastically. We illustrate this with the plot in Fig. 17. The green regions are excluded for a Higgs mass outside the range of $124-128 \mathrm{GeV}$. The gravitino mass is pushed to values far beyond $10 \mathrm{TeV}$, and for the benchmark point shown here, the gluino mass is $3 \mathrm{TeV}$, beyond current LHC reach. ${ }^{5}$ The scheme leads to some version of "hidden SUSY" that will be difficult to test at LHC. One reason is the high value of $m_{3 / 2}$ (forced upon us by the high value of the Higgs mass) another one the compressed gaugino spectrum that hides SUSY even in the cases where the gluino is accessible to production at the LHC. With a compressed spectrum the mass difference between the gluino and the lightest neutralino (LSP) is smaller than in the standard case.

\footnotetext{
$\overline{5 \text { Lower values }}$ for gluino masses are possible, as will shall discuss later.
} 


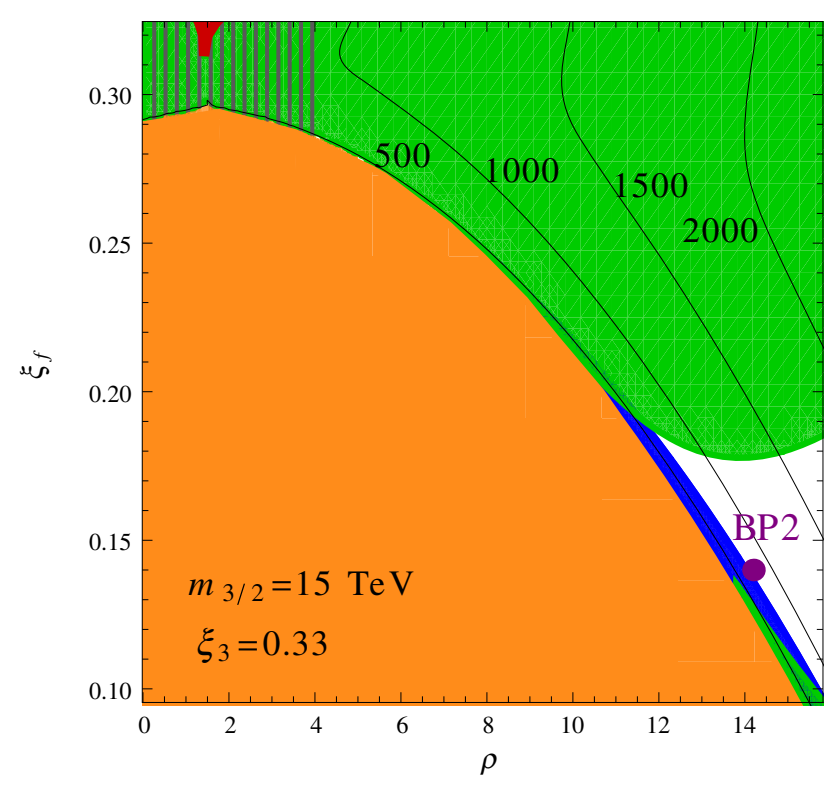

Fig. 17 Parameter scan for a benchmark model with a gluino mass of $3 \mathrm{TeV}$. The green regions are inconsistent with a Higgs mass in the range of $124-128 \mathrm{GeV}$ (for details see [64]). The hatched region indicates the reach of direct searches for SUSY (as of summer 2012). It illustrates that limits of direct searches are still weak

In the decay of the gluino the LSP thus will have less kinetic energy and this reduces the "missing energy" signal of the escaping LSP. This poses a severe challenge for the LHC as we shall see in more detail in the next subsection. The LSP is the prime candidate for cold dark matter in the universe. Within the general scenario the quest for a correct thermal relic density rules out a large part of the parameter space [65]. Still the parameter space of the scheme is sufficiently large to be consistent with all presently known experimental and cosmological constraints. We need more experimental results to clarify the situation.

\subsection{Precision supersymmetry}

Let us therefore consider a special corner of the parameter space that is theoretically well motivated. There are two strong arguments for supersymmetry:

- solution to the electroweak hierarchy problem

- gauge coupling unification.

The latter one is motivated by the evolution of gauge couplings in the MSSM as well as theoretical arguments from string theory that only provides one fundamental coupling. It might thus be interesting to take these arguments as serious as possible and demand precision gauge coupling unification (PGU) within a scheme of minimal fine tuning [68]. The evolution of gauge coupling is given by

$\frac{1}{g_{i}^{2}\left(M_{\mathrm{GUT}}\right)}=\frac{1}{g_{i}^{2}\left(M_{Z}\right)}-\frac{b_{i}^{\mathrm{MSSM}}}{8 \pi^{2}} \ln \left(\frac{M_{\mathrm{GUT}}}{M_{Z}}\right)+\frac{1}{g_{i, \mathrm{Thr}}^{2}}$

We assume that the threshold corrections vanish at the high scale. Thus they come exclusively from the MSSM spectrum

$\frac{1}{g_{i, \mathrm{Thr}}^{2}}=\frac{b_{i}^{\mathrm{MSSM}}-b_{i}^{\mathrm{SM}}}{8 \pi^{2}} \ln \left(\frac{M_{\mathrm{SUSY}}}{M_{Z}}\right)$

with the SUSY breakdown scale $M_{\text {SUSY }}$. To quantify precision gauge unification (PGU) we define

$\epsilon_{3}=\frac{g_{3}^{2}\left(M_{\mathrm{GUT}}\right)-g_{1,2}^{2}\left(M_{\mathrm{GUT}}\right)}{g_{1,2}^{2}\left(M_{\mathrm{GUT}}\right)}$

and demand $\epsilon_{3}=0$. The relation between $\epsilon_{3}$ and $M_{\text {SUSY }}$ is given in Fig. 18, and $\epsilon_{3}=0$ leads to $M_{\text {SUSY }} \sim 2 \mathrm{TeV}$. We now have to determine $M_{\text {SUSY }}$ in a given model. In the case that all the supersymmetric partners have the same mass $\mathrm{M}$, then $M_{\text {SUSY }}=M$. For non- universal masses we have an effective scale

$M_{\text {SUSY }} \sim \frac{m_{\widetilde{W}}^{32 / 19} m_{\widetilde{h}}^{12 / 19} m_{H}^{3 / 19}}{m_{\widetilde{g}}^{28 / 19}} X_{\text {sfermion }}$

where $m_{\widetilde{W}}, m_{\widetilde{h}}, m_{H}$ and $m_{\widetilde{g}}$ denote the mass of the wino, the higgsino, the heavy Higgs and the gluino, respectively. Within this class of models considered here the effect of sfermions is small [68]: $X_{\text {sfermion }} \approx 1$. Let us first examine the value of $M_{\text {SUSY }}$ in the so-called CMSSM (i.e. the MSSM with universal gaugino masses $m_{1 / 2}$ at the GUT scale). At the weak scale we would have the gaugino mass ratio [67]

$M_{1}: M_{2}: M_{3}=1: 2: 6$

and the effective SUSY scale reads

$M_{\mathrm{SUSY}} \simeq 0.3\left(m_{\widetilde{h}}^{12} m_{1 / 2}^{4} m_{H}^{3}\right)^{1 / 19} X_{\text {sfermion }}$

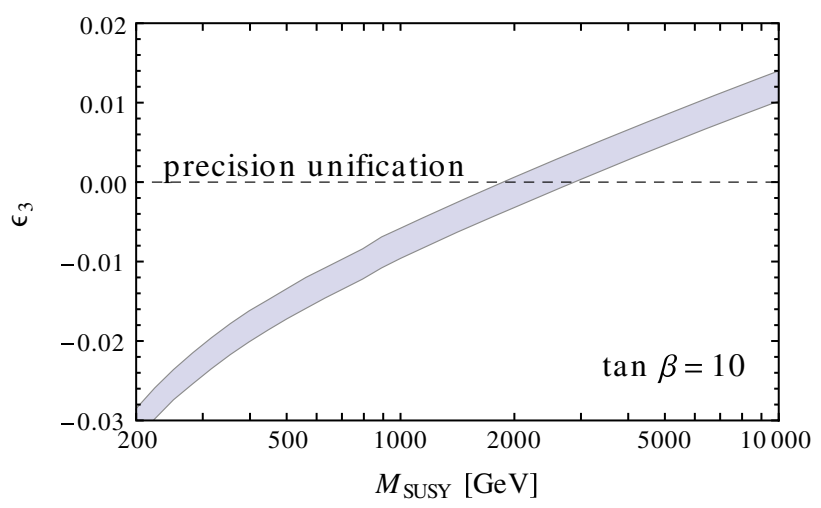

Fig. 18 Precision gauge unification $\left(\epsilon_{3}=0\right)$ requires a SUSY scale around $2-3 \mathrm{TeV}[68]$ 
To reach a scale $M_{\text {SUSY }} \approx 2 \mathrm{TeV}$ as required by precision gauge unification this leads to a large higgsino mass

$m_{\tilde{h}} \simeq 20 \mathrm{TeV} \times\left(\frac{\mathrm{TeV}}{m_{1 / 2}}\right)^{1 / 3}\left(\frac{\mathrm{TeV}}{m_{H}}\right)^{1 / 4}$

As $m_{\tilde{h}} \sim \mu \sim 20 \mathrm{TeV}$ we will have a severe fine-tuning problem. Since we require a minimal amount of fine tuning we shall discard the CSSM as a natural framework to obtain precision gauge unification. Are there alternatives?

The string-inspired pattern of the SUSY breaking scheme discussed earlier exhibits mirage mediation and a compressed gaugino mass spectrum. The gaugino masses can be written as

$M_{i}=\frac{m_{3 / 2}}{16 \pi^{2}}\left(\varrho+b_{i}^{\mathrm{MSSM}} g^{2}\right)$

where $m_{3 / 2}$ denotes the gravitino mass and $\varrho$ parametrizes the modulus mediated contribution to gaugino masses. This leads to

$M_{1}: M_{2}: M_{3}=(\varrho+3.3): 2(\varrho+0.5): 6(\varrho-1.5)$

and exhibits a strong compression of gaugino masses for small $\varrho$ (and even an unphysical region where the gluino is the lightest gaugino). Now let us have another look at $M_{\text {SUSY }}$ :

$M_{\text {SUSY }} \sim \frac{m_{\widetilde{W}}^{32 / 19} m_{\widetilde{h}}^{12 / 19} m_{H}^{3 / 19}}{m_{\widetilde{g}}^{28 / 19}}$

Key observation is the fact that an increase of the gluino mass reduces $M_{\text {SUSY }}$. In the case of a compressed spectrum with wino and gluino masses of similar size we obtain PGU (i.e. $M_{\text {SUSY }} \sim 2 \mathrm{TeV}$ ) for a smaller value of $\mu$ and therefore less fine tuning. This is illustrated in Fig. 19.

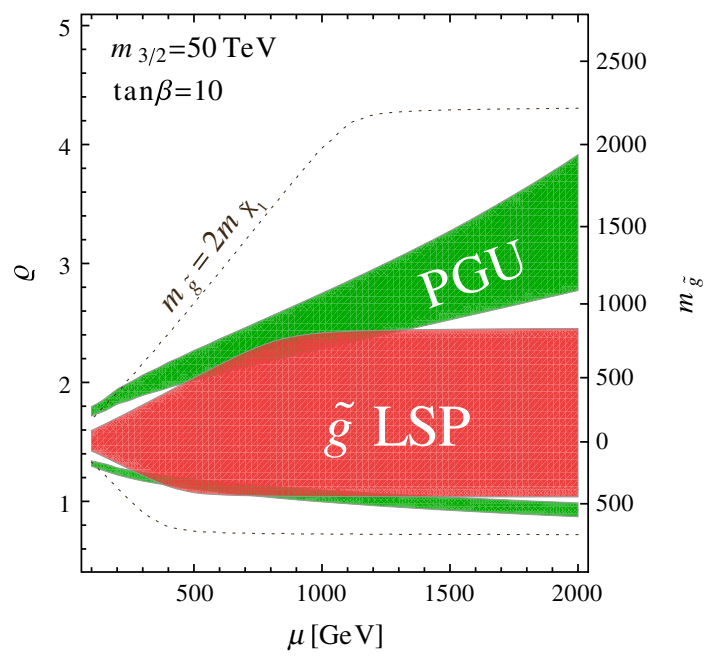

Fig. 19 Gluino mass versus $\mu$ for various values of $\varrho$. Green regions are consistent with small $\varrho$ and an ultra-compressed spectrum of gaugino masses satisfying PGU [68]

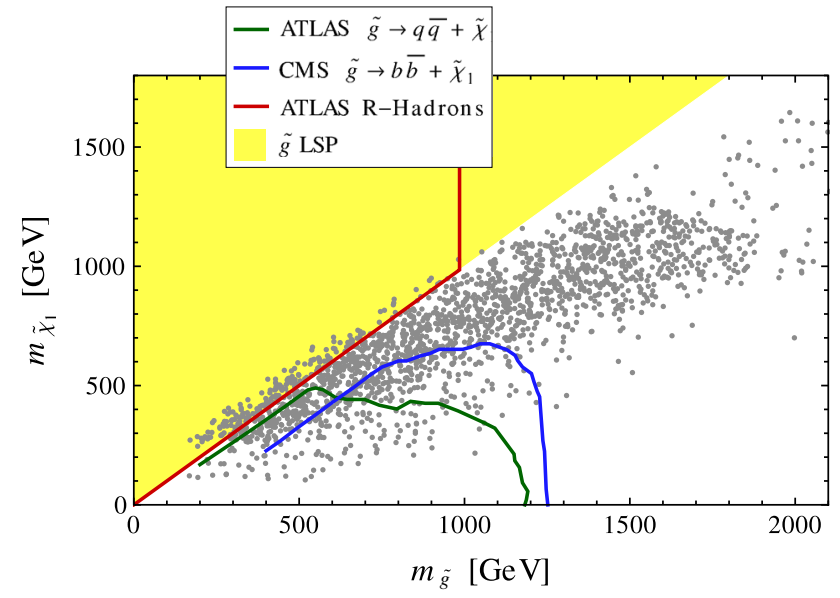

Fig. 20 Neutralino versus gluino mass for a sample of benchmark models that satisfy PGU with a compressed gaugino mass spectrum [68]. Current limits from LHC are still weak

The green regions are consistent with PGU. We see that rather small values of $m_{\tilde{g}}$ and $\mu \sim m_{\tilde{h}}$ allow for PGU, both in the kinematic reach of the LHC. Known results from LHC might thus constrain the models and even rule them out. To check the validity of the model, we have generated a large data sample [68] with random input parameters that lead to successful PGU. In Fig. 20 we provide a scatter plot of those parameters and include present limits from LHC (ATLAS search [69] and CMS results for b-jets and missing energy [70]; for a detailed discussion see [68]). Present LHC limits are weak. Only a small part of the parameter space is ruled out. The strongly compressed gaugino mass spectrum makes it difficult to detect even rather light gluinos. It will be interesting to see the discovery potential for the LHC in the next run, as large parts of the parameter space are kinematically accessible.

More restrictions on the model could come from the requirement of the correct thermal relic abundance of the lightest supersymmetric particle (LSP) as a candidate for cold dark matter. Prime candidates are the bino and the higgsino. Due to its small annihilation cross section, the bino density from thermal production typically exceeds the observed dark matter density by far. Higgsinos, on the other hand, undergo efficient annihilation into third generation quarks or gauge bosons, and co-annihilations with the charged higgsino further enhance their cross section. Hence, the relic density of a higgsino LSP might typically be below the dark matter density. In mirage mediation, the gaugino masses are nonuniversal at the high scale and lead to highly compressed gaugino spectrum at the weak scale as a consequence of PGU. This enhances the possibility for co-annihilations which is favorable for the dark matter density. In Fig. 21 we compare the neutralino relic density for the sample points with or without imposing PGU. 


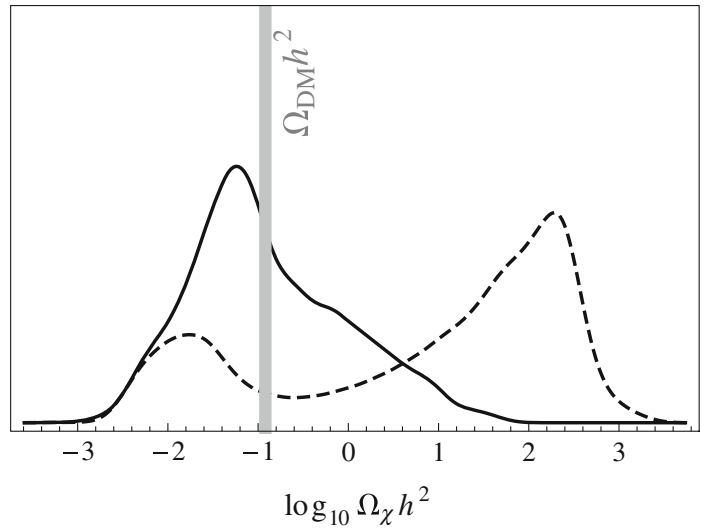

Fig. 21 Distribution of the thermal neutralino relic density for the benchmark sample with (solid) or without (dashed) the assumption of precision gauge coupling unification [68]

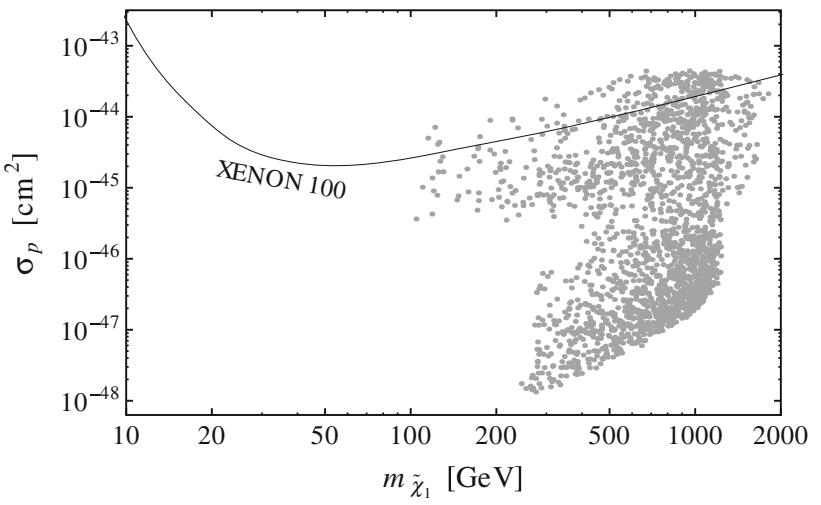

Fig. 22 Neutralino proton cross section for the benchmark points with successful PGU [68]. The current limit from XENON100 direct dark matter search is shown. The latter is only applicable if the lightest neutralino accounts for all dark matter of the universe

Models with PGU seem to be able to accomodate the correct relic density rather easily. Reach and limits from direct detection of these dark matter candidates are shown in Fig. 22.

Future experiments might probe a significant part of the parameter space of the models.

\section{Conclusion}

As we have seen it is a long way from string theory via the MSSM to LHC physics. To test these ideas we need consistent string theory constructions that allow explicit determination of spectrum and interactions to be confronted with the data. At this point only the models of the heterotic MiniLandscape satisfy both criteria. Given these models we can try to extract some generic properties from the successful MSSM candidates. Essentially these are lessons originated from the geographic localization of fields in compactified extra-dimensional space. A coherent picture emerges, Higgs and top multiplets live in the bulk. This provides a solution to the $\mu$-problem with an R-symmetry as well as a large value for the Yukawa coupling of the top quark (to be consistent with so-called gauge-Yukawa unification). The multiplets of the first two families are located at fixed points in extradimensional space. They enjoy enhanced gauge- and discrete symmetries that alleviate the flavor problem. A slight breakdown of these symmetries provides a small parameter (originated from a Fayet-Iliopoulos term) that could explain the hierarchies of quark and lepton masses as well as the $\mu$-parameter. We expect these properties (derived from the heterotic string theory) to be of more general validity and should also manifest themselves in constructions based on type I, type II, M- and F-theory.

In the discussion of SUSY breakdown we can identify a rather generic scheme: mirage mediation. It has been found both in Type IIB and heterotic theory and is a consequence of the mechanism to obtain a small value of the vacuum energy (compared to the scale of the gravitino mass). The scheme is characterized by two scales for the soft terms separated by a factor $\log \left(M_{\text {Planck }} / m_{3 / 2}\right)$. Gaugino masses and A- parameters tend to be at the $\mathrm{TeV}$ scale, while gravitino mass and scalar masses are generically at a higher scale. A second characteristic property of the mirage scheme is the possibility of a compressed spectrum of the gaugino masses as shown in Fig. 23. It leads to hidden SUSY at the LHC and allows for the correct thermal relic density of the LSP dark matter candidate.

Within the heterotic scheme we could identify another important result concerning scalar masses, determined by the localization properties of the corresponding fields with a potential protection through extended supersymmetry. Localized fields as e.g. the scalar partners of quarks and

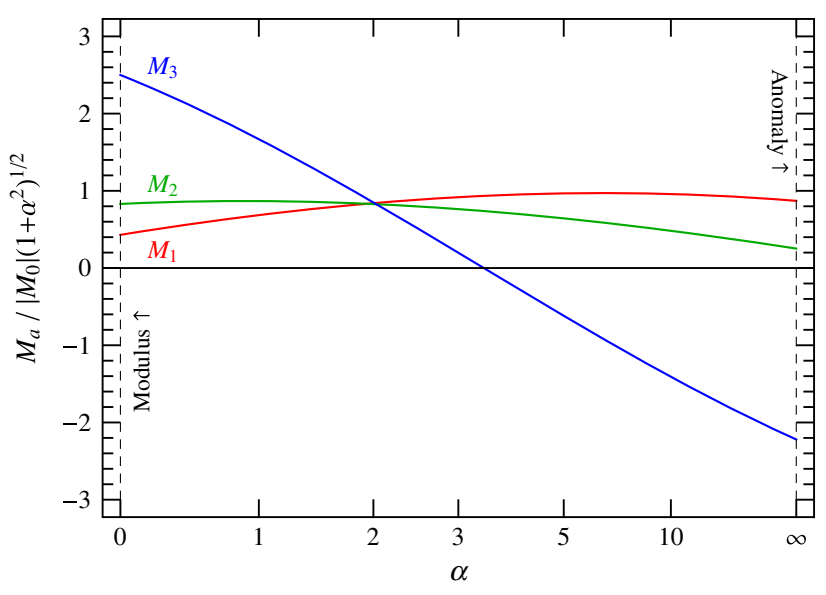

Fig. 23 Gaugino masses as a function of $\alpha$, the ratio of anomaly to modulus mediated contributions ( $\alpha$ is inversely proportional to $\varrho$ defined earlier) [62]. We clearly see the possibility of an ultra-compressed spectrum around $\alpha=2$ 
leptons of the first two families only feel $N=1$ SUSY and would be as heavy as the gravitino. Fields at fixed tori or the bulk feel a hidden $N=2$ or $N=4$ SUSY and have suppressed masses comparable to those of the gaugino masses. It is this interplay of symmetries that leads to very specific properties of the spectrum of superpartners. The scheme is still consistent with all known experimental data. A large part of the parameter space is within the kinematical reach of the LHC at $14 \mathrm{TeV}$. The next run of the LHC might hopefully test these ideas.

Open Access This article is distributed under the terms of the Creative Commons Attribution License which permits any use, distribution, and reproduction in any medium, provided the original author(s) and the source are credited.

Funded by $\mathrm{SCOAP}^{3}$ / License Version CC BY 4.0.

\section{References}

1. G. Aad et al., ATLAS Collaboration. Phys. Lett. B 716, 1 (2012). [arXiv:1207.7214 [hep-ex]]

2. S. Chatrchyan et al., CMS Collaboration. Phys. Lett. B 716, 30 (2012). [arXiv:1207.7235 [hep-ex]]

3. M.B. Green, J.H. Schwarz, Phys. Lett. B 149, 117 (1984)

4. H.P. Nilles, S. Ramos-Sanchez, M. Ratz, P.K.S. Vaudrevange, Eur. Phys. J. C 59, 249 (2009). [arXiv:0806.3905 [hep-th]]

5. A.N. Schellekens, Life at the Interface of Particle Physics and String Theory. [arXiv:1306.5083 [hep-ph]].

6. K.-S. Choi, J.E. Kim, Quarks and leptons from orbifolded superstring. Lect. Notes Phys. 696, 1 (2006)

7. L.E. Ibanez, A.M. Uranga, String Theory and Particle Physics: An Introduction to String Phenomenology (University Press, Cambridge, UK, 2012), p. 673

8. R. Blumenhagen, B. Kors, D. Lust, S. Stieberger, Phys. Rept. 445, 1 (2007). [arXiv:hep-th/0610327]

9. O. Lebedev, H.P. Nilles, S. Raby, S. Ramos-Sánchez, M. Ratz, P.K.S. Vaudrevange, A. Wingerter, Phys. Lett. B645, 88 (2007). [arXiv:hep-th/0611095]

10. O. Lebedev, H.P. Nilles, S. Raby, S. Ramos-Sánchez, M. Ratz, P.K.S. Vaudrevange, A. Wingerter, Phys. Rev. D77, 046013 (2007). [arXiv:0708.2691 [hep-th]]

11. O. Lebedev, H.P. Nilles, S. Ramos-Sanchez, M. Ratz, P.K.S. Vaudrevange, Phys. Lett. B 668, 331 (2008). [arXiv:0807.4384 [hepth]]

12. H.P. Nilles, Five golden rules for superstring phenomenology, [arXiv:hep-th/0410160]

13. S. Förste, H.P. Nilles, P.K.S. Vaudrevange, A. Wingerter, Phys. Rev. D70, 106008 (2004). [arXiv:hep-th/0406208]

14. D.J. Gross, J.A. Harvey, E.J. Martinec, R. Rohm, Nucl. Phys. B 256, 253 (1985)

15. D.J. Gross, J.A. Harvey, E.J. Martinec, R. Rohm, Nucl. Phys. B267, 75 (1986)

16. P. Candelas, G.T. Horowitz, A. Strominger, E. Witten, Nucl. Phys. B 258, 46 (1985)

17. L.J. Dixon, J.A. Harvey, C. Vafa, E. Witten, Nucl. Phys. B 261, 678 (1985)

18. L.J. Dixon, J.A. Harvey, C. Vafa, E. Witten, Nucl. Phys. B 274, 285 (1986)

19. L.E. Ibáñez, H.P. Nilles, F. Quevedo, Phys. Lett. B 187, 25 (1987)

20. L.E. Ibáñez, J.E. Kim, H.P. Nilles, F. Quevedo, Phys. Lett. B 191, $282(1987)$
21. A.E. Faraggi, Nucl. Phys. B 387, 239 (1992). [arXiv:hep-th/ 9208024]

22. F. Gmeiner, G. Honecker, JHEP 0807, 052 (2008). [arXiv:0806. 3039 [hep-th]]

23. G. Honecker, J. Vanhoof, Fortsch. Phys. 60, 1050 (2012). [arXiv: 1201.5872 [hep-th]]

24. V. Braun, Y.H. He, B.A. Ovrut, T. Pantev, Phys. Lett. B 618, 252 (2005). [arXiv:hep-th/0501070]

25. V. Bouchard, R. Donagi, Phys. Lett. B 633, 783 (2006). [arXiv: hep-th/0512149]

26. L.B. Anderson, J. Gray, A. Lukas, E. Palti, JHEP 1206, 113 (2012). [arXiv:1202.1757 [hep-th]]

27. L.B. Anderson, J. Gray, A. Lukas, E. Palti, PoS CORFU 2011, 096 (2011)

28. R. Donagi, M. Wijnholt, Adv. Theor. Math. Phys. 15, 1237 (2011) [arXiv:0802.2969 [hep-th]]

29. C. Beasley, J.J. Heckman, C. Vafa, JHEP 0901, 058 (2009). [arXiv: 0802.3391 [hep-th]]

30. T.P.T. Dijkstra, L.R. Huiszoon, A.N. Schellekens, Nucl. Phys. B 710, 3 (2005). [arXiv:hep-th/0411129]

31. P. Anastasopoulos, T.P.T. Dijkstra, E. Kiritsis, A.N. Schellekens, Nucl. Phys. B 759, 83 (2006). [arXiv:hep-th/0605226]

32. T. Kobayashi, S. Raby, R.J. Zhang, Phys. Lett. B 593, 262 (2004). [arXiv:hep-ph/0403065]

33. T. Kobayashi, S. Raby, R.J. Zhang, Nucl. Phys. B 704, 3 (2005)

34. W. Buchmüller, K. Hamaguchi, O. Lebedev, M. Ratz, Phys. Rev. Lett. 96, 121602 (2006). [arXiv:hep-ph/0511035]

35. W. Buchmüller, K. Hamaguchi, O. Lebedev, M. Ratz, Nucl. Phys. B 785, 149 (2007). [arXiv:hep-th/0606187]

36. M. Blaszczyk, S. Nibbelink, Groot, M. Ratz, F. Ruehle, M. Trapletti, P.K.S. Vaudrevange. Phys. Lett. B 683, 340 (2010). [arXiv:0911.4905 [hep-th]]

37. J.E. Kim, B. Kyae, Flipped SU(5) from Z(12-I) orbifold with Wilson line (2006). [arXiv:hep-th/0608085]

38. J.E. Kim, J.H. Kim, B. Kyae, JHEP 06, 034 (2007). [arXiv:hep-ph/ 0702278]

39. D.K.M. Pena, H.P. Nilles, P.-K. Oehlmann, JHEP 1212, 024 (2012). [arXiv:1209.6041 [hep-th]]

40. S.G. Nibbelink, O. Loukas, [arXiv:1308.5145 [hep-th]]

41. C.D. Froggatt, H.B. Nielsen, Nucl. Phys. B 147, 277 (1979)

42. R. Kappl, H.P. Nilles, S. Ramos-Sanchez, M. Ratz, K. SchmidtHoberg, P.K.S. Vaudrevange, Phys. Rev. Lett. 102, 121602 (2009). [arXiv:0812.2120 [hep-th]]

43. F. Brummer, R. Kappl, M. Ratz, K. Schmidt-Hoberg, JHEP 1004, 006 (2010). [arXiv:1003.0084 [hep-th]]

44. H.M. Lee, S. Raby, M. Ratz, G.G. Ross, R. Schieren, K. SchmidtHoberg, P.K.S. Vaudrevange, Phys. Lett. B 694, 491 (2011). [arXiv: 1009.0905 [hep-ph]]

45. L.E. Ibanez, H.P. Nilles, F. Quevedo, Phys. Lett. B 192, 332 (1987)

46. A. Font, L.E. Ibanez, H.P. Nilles, F. Quevedo, Nucl. Phys. B 307 109 (1988). [Erratum-ibid. B 310 (1988) 764]

47. J.A. Casas, C. Muñoz, Phys. Lett. B 306, 288 (1993). [arXiv: hep-ph/9302227]

48. T. Kobayashi, H.P. Nilles, F. Plöger, S. Raby, M. Ratz, Nucl. Phys. B 768, 135 (2007). [arXiv:hep-ph/0611020]

49. H.P. Nilles, M. Ratz, P.K.S. Vaudrevange, Fortsch. Phys. 61, 493 (2013). [arXiv:1204.2206 [hep-ph]]

50. H.P. Nilles, Phys. Lett. B 115, 193 (1982)

51. S. Ferrara, L. Girardello, H.P. Nilles, Phys. Lett. B 125, 457 (1983)

52. J.P. Derendinger, L.E. Ibáñez, H.P. Nilles, Phys. Lett. B 155, 65 (1985)

53. M. Dine, R. Rohm, N. Seiberg, E. Witten, Phys. Lett. B 156, 55 (1985)

54. O. Lebedev, H.P. Nilles, S. Raby, S. Ramos-Sánchez, M. Ratz, P.K.S. Vaudrevange, A. Wingerter, Phys. Rev. Lett. 98, 181602 (2007). [arXiv:hep-th/0611203] 
55. K. Choi, A. Falkowski, H.P. Nilles, M. Olechowski, S. Pokorski, JHEP 0411, 076 (2004). [arXiv:hep-th/0411066]

56. K. Choi, A. Falkowski, H.P. Nilles, M. Olechowski, Nucl. Phys. B 718, 113 (2005). [arXiv:hep-th/0503216]

57. O. Lebedev, H.P. Nilles, M. Ratz, Phys. Lett. B 636, 126 (2006). [arXiv:hep-th/0603047]

58. V. Nilles, J. Plank, CCR, 42, 736 (2012)

59. O. Lebedev, H.P. Nilles, M. Ratz, A Note on fine-tuning in mirage mediation. [arXiv:hep-ph/0511320]

60. O. Loaiza-Brito, J. Martin, H.P. Nilles, M. Ratz, $\log (\mathrm{M}(\mathrm{Pl}) /$ m(3/2)). AIP Conf. Proc. 805, 198 (2006). [arXiv:hep-th/0509158]

61. O. Lebedev, V. Lowen, Y. Mambrini, H.P. Nilles, M. Ratz, JHEP 0702, 063 (2007). [arXiv:hep-ph/0612035]

62. V. Löwen, H.P. Nilles, Phys. Rev. D77, 106007 (2008). 0802.1137

63. V. Lowen, H.P. Nilles, Nucl. Phys. B 827, 337 (2010). [arXiv:0907. 4983 [hep-ph]]

64. S. Krippendorf, H.P. Nilles, M. Ratz, M.W. Winkler, Phys. Lett. B 712, 87 (2012). [arXiv:1201.4857 [hep-ph]]
65. M. Badziak, S. Krippendorf, H.P. Nilles, M.W. Winkler, JHEP 1303, 094 (2013). [arXiv:1212.0854 [hep-ph]]

66. S. Heinemeyer, W. Hollik, D. Stockinger, A.M. Weber, G. Weiglein, Pramana 69, 783 (2007). [arXiv:hep-ph/0611371]

67. K. Choi, H.P. Nilles, JHEP 0704, 006 (2007). [arXiv:hep-ph/ 0702146 [HEP-PH]]

68. S. Krippendorf, H.P. Nilles, M. Ratz, M.W. Winkler, Phys. Rev. D 88, 035022 (2013). [arXiv:1306.0574 [hep-ph]]

69. ATLAS Collaboration, Search for squarks and gluinos with the ATLAS detector using final states with jets and missing transverse momentum and $5.8 \mathrm{fb}^{-1}$ of $\sqrt{s}=8 \mathrm{TeV}$, ATLAS-CONF-2012-109, ATLAS-COM-CONF-2012-140

70. CMS Collaboration, Search for Supersymmetry in pp collisions at $8 \mathrm{TeV}$ in events with a single lepton, multiple jets and b-tags, CMS-PAS-SUS-13-007 